\title{
Cost evaluation of innovative offers using detailed equipment, process and network modeling languages
}

\author{
Koen Casier, Marlies Van der Wee, Sofie Verbrugge \\ Ghent University - iMinds, Gaston Crommenlaan 8 box 201, 9050 Ghent, Belgium \\ \{Koen.casier, marlies.vanderwee, sofie.verbrugge\}@intec.ugent.be
}

\begin{abstract}
The fast evolution of networks, techniques and tools makes designing the business case for a new high tech customer offer a collaborative task, in which multiple specialized firms each provide their own expertise to the offer, making it as such the most advanced, yet cost-effective on the market. While in the past, the design of such a business case was made using the "back of an envelope" calculation, the complexity such collaborative effort entails, increases the need for specific tools for modeling the firms' interaction without losing the specifics of the cost-benefit models for the individual partners. To contribute to the effective design of collaborative business cases, this paper will describe a scalable multi-level quantification approach, while focusing on specific modeling languages for detailed cost modeling for the different partners: equipment modeling, process modeling and network modeling.
\end{abstract}

Keywords: cost modelling, equipment, process, network, modelling language

\section{INTRODUCTION}

Novel business model innovations for high tech customer offers frequently require the collaboration of multiple firms, each with their own specializations. As such, a need rises for the development of tools that allow modeling the entire business case across the different partners involved, without losing the details for each firm (or part of the offer) individually. Furthermore, investment decisions are currently based on dedicated calculations and models, designed at hoc and as such with limited replicability. A second need can thus be identified by indicating the potential benefits of tools that allow dedicated, detailed, but re-usable modeling for specific smaller-scale cost modeling tasks.

The BEMES (Business Modeling and Simulation) approach developed under the Fiware umbrella, is a tool that addresses exactly these two challenges identified above. The high-level business modeling allows modeling interactions between different actors, while the business calculator offers specific modeling languages that can be used to draw out easily interpretable cost models for each part of the business case offer. As such the BEMES tool adds intuitive modeling approaches to the field of business modeling and techno-economic research, and provides reliable tools for translating these models into cost structures. While previous work [1] focused on describing the high-level overview of both the business modeling, business calculator and their interaction though value streams and revenue models, this paper will focus on the different specific modeling languages that were developed within the business calculator [2].

As such, the remainder of this paper is structured according to the different modeling languages: section 2 describes the Physical Network Modeling Notation (PNMN), section 3 focuses on the Equipment Cost Modeling Notation (ECMN), while the Business Process Modeling Notation (BPMN) is detailed in section 4. Each modeling language aims at (1) graphically designing the model, (2) calculating amounts - of equipment to be installed, process tasks to be executed, or $\mathrm{km}$ trench to be dug - and (3) translating these amounts in cost (investment) requirements over time. Each section will thus touch upon these three goals. Finally, section 5 summarizes the paper and gives directions for future work and application domains for the BEMES tool.

\section{PNMN: Network Modeling}

Fast estimation of infrastructure deployment costs based on analytical approximations

When deploying a new infrastructure, the largest part of the investment cost is taken up by the installation of the outside plant, i.e. the manual labor for trenching and installation of the cables. Although real deployments need a detailed rollout plan indicating the exact amount of cables and trench distance, in the business modeling phase, a fast, yet reliable estimation is preferable in the strategic investment analysis. In this phase many alternative installations will be compared to each other in search for a set of scenarios for instance in which costs are reduced, revenues increased, risks contained. In the example given below (Fig. 1), an FTTH access network with two levels of hierarchy is schematically represented. In Fig. 1a, the feeder section (in between central office and street cabinet) is modeled using direct connections, analytically approached as a diagonal tree structure, while Fig. 1b indicates the distribution section from the street cabinet to individual houses, which will typically follow the sidewalks and which is analytically approached as a uniform double street length model. 


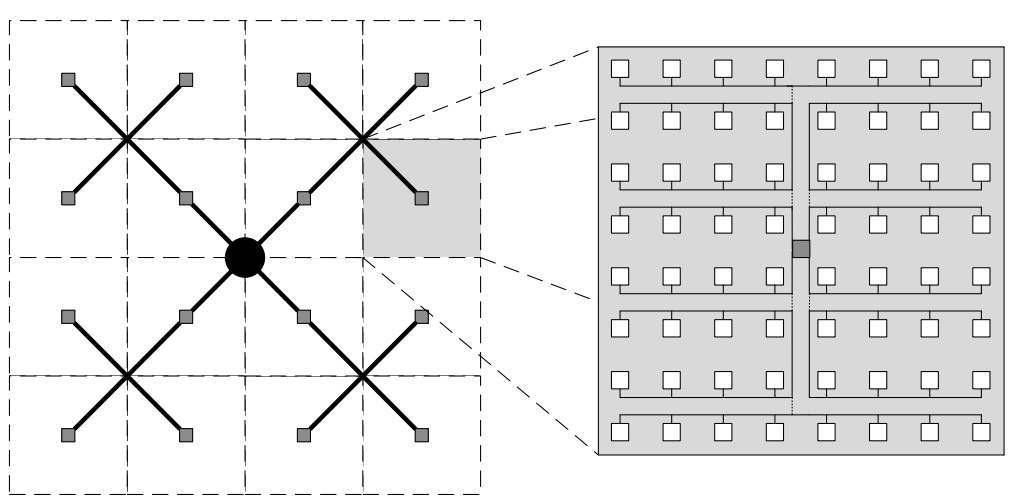

Fig. 1: Schematic representation of a FTTH access network: direct feeder section from central office to street cabinet (left), double street model distribution section from street cabinet to houses (right).

The graphical user interface for PNMN allows the user to choose the deployment model (not limited to the choices presented above, see [3]), and indicate the relevant parameters: the population density and the surface in $\mathrm{km}^{2}$. The model then provides the trenching (or aerial deployment) distance, as well as the total cable and fiber length. As the model is structured hierarchically, it is possible to use different deployment models for different parts of the network (see example above), and the level of hierarchies is unlimited.

\section{ECMN: Equipment Coupling Modeling Notation}

\section{Modeling granular equipment placement over time}

The second modeling language developed in BEMES is ECMN: Equipment Cost Modeling Notation. ECMN draws from a hierarchical structure that allows determining the amount and cost of equipment to be installed. By installing only the equipment that is needed at each point in time (based on the amounts of drivers), the costs of equipment are spread out and the investing firm receives a direct payoff that can be used to pay back the investment in equipment. An example for the equipment tree designed for filling a central office for a P2P FTTH deployment can be found in Fig. 2. The central office in this case can be linked to the output of the previous network infrastructure calculation. The same approach can be used to dimension the costs of the street cabinet installations or any other element in the network. Clearly the same equipment coupling model can be used in dimensioning central offices, data centers, storage servers or any other equipment structure.

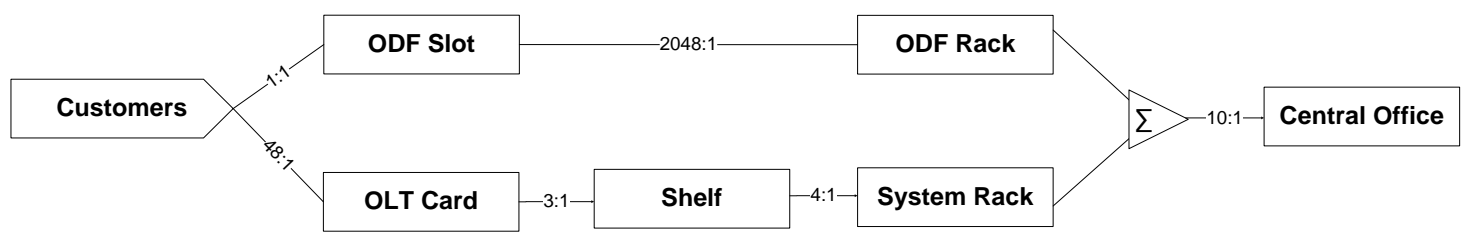

Fig. 2: Example of an ECMN tree for installation of a central office in a FTTH network

In a first step, a tree structure is graphically drawn indicating the equipment that needs to be installed in the central office, while linking them using granularity factors (i.e. one shelf can accommodate at max three OLT cards). In a second step, the needed input parameters are defined per equipment (cost, maintenance, failure rate, power consumption, etc.) and on the entire model (time functions for the drivers initiating the calculation, e.g. the amount of customers that will need to be served in each year). As these drivers are linked to the lowest equipment types in the graphical tree (customers drive the amount of ODF slots and OLT cards), they serve as such as drivers for the higher branches, thereby allowing calculations of the entire tree bottom-up, for both amounts of each equipment type, as well as costs, both initial investment (CapEx) and continuous costs (OpEx).

ECMN can of course be applied for more and different types of equipment modeling, e.g. the ratio of the different parts needed for assembling a new product based on different requirements, the equipment installed in computer rooms in schools based on the number of students, etc. Additional features allow easy identification of minimal installation sizes, switchover of equipment types over time, aggregation rules based on summation (shown in figure), maximum, average, etc. The calculations render the amounts to install in time, but also the expected cost, energy consumption, space requirements, etc. when the specifications for the equipment are completely filled. For a more detailed description of the modeling and calculation, we refer to [4]. 


\section{BPMN: Business Process Modeling Notation Using flows to determine the cost of automated or manual processes}

Finally, BEMES includes a third and final specific modeling language for the modeling of processes. BPMN (Business Process Modeling Notation) is a well-developed standard [5] based on flowchart structures. Processes can easily be translated into flowcharts and BPMN contains a rich set of modeling elements allowing to model even the more arcane process structures. Linking these flowcharts to the right cost parameters (e.g. cost per hour of manual labor), and using activity based costing [6]-[7], allows for a clear, statistical calculation of the operational process cost for a specific time horizon.

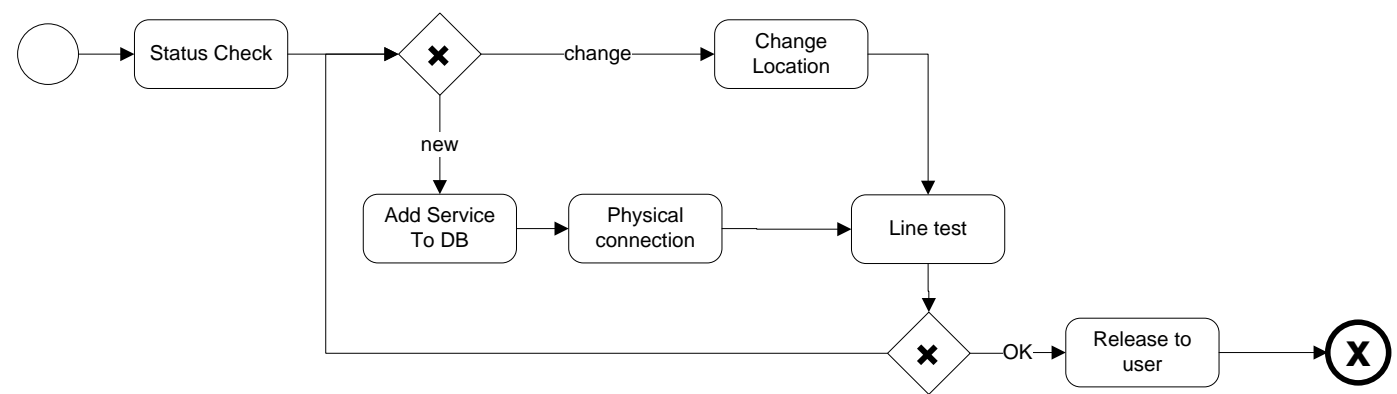

Fig. 3: Example of a BPMN process for the connection of a (new or existing) customer to a FTTH access network

An example of a BPMN process model is given in Fig. 3. This is a simplified example of a customer provisioning process, in which the customer wants to connect to the network, or change his existing connection. This process details the different steps that need to be executed in order to complete his request. BPMN already provides all flexibility to incorporate decisions and conditions by exclusive gateways (change/new customer, succeeded or failed line test $(\mathrm{OK} / \mathrm{NOK}))$ and fork or join gateways (the line test will only be started if the location has been changed or the physical connection has been formed). By inserting the right information at the different events (time to execute, needed man power, etc.), at the gateways (probability of each paths) and at the start events (number of event per time), the cost for execution of the entire process can be calculated using the activity based costing approach.

Again the operational process will be coupled to aspects of the network and of the equipment. Clearly maintenance of equipment and network infrastructure will be depending on the amount and types of equipment installed in the network. Again operational processes are not limited to telecommunication networks and can be used for modeling any kind process-based work (with manual and/or automated tasks).

\section{CONCLUSIONS}

The design of new products, the development of new services, and the deployment of new networks all incorporate a significant level of risk. Since technical evolutions furthermore drive specialization of firms, collaboration is an extra factor to take into account in these new business cases. Therefore, before starting the actual production or rollout, a need arises for a detailed modeling and understanding of the different costs that are included, and this for the different partners involved.

A first step in this process consists of gathering all partners and their information on the problem at hand. When deploying a new network, the network installation, network operation, network maintenance, customer acquisition and provisioning, etc. will be required and different partners (e.g. firms, municipalities) will interact (either in cooperation or competition). The business model shows which partners to contact and which cost factors to take into account, but this is merely a starting point. A detailed cost modeling is required in order to get a good feel of the costs, but also get a look at the potential risks, alternative scenarios, etc. It is important to build such cost models based on expert knowledge and translate this into a model everybody can intuitively understand and which follows naturally the actual cost structure. As such several domain specific cost modeling languages should be used and combined into one overarching model.

This paper details the specific modeling languages that are part of the BEMEs approach, which combines detailed cost modeling with higher-level business modeling to get a concrete overview of the entire business case results. Three modeling languages were described, each focusing on a specific standardization of a typical cost part in a business case. The first language allows modeling the trenching and cable distance for newly-to-deploy networks, and is based on a set of analytical models. ECMN, the second language, allows calculating the amount 
and cost of equipment placement by setting up a granular tree structure. Finally, BPMN uses process flowcharts to estimate the cost of specific processes.

Using these standardized modeling languages will improve the reusability of the models, increase collaboration opportunities, and will allow for modeling more complex applications.

\section{ACKNOWLEDGEMENTS}

The authors have received funding from the Agency for Innovation by Science and Technology in Flanders, Belgium, and the BEMES project is part of the FI-WARE: Future Internet Core Platform European project (grant-nr. 285248).

\section{REFERENCES}

[1] Casier, K., Van der Wee, M., Verbrugge, s., Ranaivoson, H., Reynders, C., Coenen, T. Multi-level Business Modeling and Simulation, Symposium on Business Modeling and Software Development. BMSD, June 2014.

[2] The Business Calculator Open Specifications http://forge.fi-ware.org/plugins/mediawiki/wiki/fiware/ index.php/FIWARE.ArchitectureDescription.Apps.BusinessCalculator

[3] Attila Mitcsenkov, Miroslaw Kantor, Koen Casier, Bart Lannoo, Krzysztof Wajda, Jiajia Chen, Lena Wosinska, Geometric versus Geographic Models for the Estimation of an FTTH Deployment, Telecom Systems Journal special issue, May 2016 (accepted)

[4] Van der Wee M., et al., A modular and hierarchically structured techno-economic model for FTTH deployments. Comparison of technology and equipment placement as function of population density and number of flexibility points, ONDM 2012

[5] Object Management Group/Business Process Management Initiative, http://www.bpmn.org/

[6] Kaplan R.S., Anderson S.: "Time-Driven Activity-Based Costing," Harvard Business Review, 2004, vol. 82, no. 11, pp. 131-138.

[7] Casier K.,, Techno-Economic Evaluation of a Next Generation Access Network Deployment in a Competitive Setting, PhD degree at the Faculty of Engineering of the Ghent University obtained, October 8th 2009 